\title{
Fatty acid-induced mitochondrial uncoupling in adipocytes is not a promising target for treatment of insulin resistance unless adipocyte oxidative capacity is increased
}

\author{
K. N. Frayn • D. Langin $\cdot$ F. Karpe
}

Received: 24 October 2007 / Accepted: 25 October 2007 / Published online: 21 December 2007

(C) Springer-Verlag 2007

\begin{abstract}
The release of fatty acids from white adipose tissue is regulated at several levels. We have examined the suggestion that fatty acid release might be diminished by upregulation of mitochondrial fatty acid oxidation in the adipocyte, through increasing mitochondrial uncoupling. The intrinsic oxidative capacity of white adipose tissue is low, and older studies suggest that there is little fatty acid oxidation in white adipocytes, human or rodent. We have examined data on fatty acid metabolism and $\mathrm{O}_{2}$ consumption in human white adipose tissue in vivo, and conclude that increasing fatty acid oxidation within the oxidative capacity of the tissue would produce only small changes (a few percent) in fatty acid release. The major locus of control of fatty acid release beyond the stimulation of lipolysis is the pathway of fatty acid esterification, already probably targeted by the thiazolidinedione insulin-sensitising agents.
\end{abstract}

K. N. Frayn · F. Karpe $(\bowtie)$

Oxford Centre for Diabetes, Endocrinology and Metabolism,

University of Oxford, Churchill Hospital,

Oxford OX3 7LJ, UK

e-mail: fredrik.karpe@ocdem.ox.ac.uk

D. Langin

Inserm U858, Institut de Médecine Moléculaire de Rangueil, Laboratoire de recherches sur les obésités,

Toulouse, France

D. Langin

Université Paul Sabatier, Institut Louis Bugnard,

IFR31, Toulouse, France

D. Langin

CHU de Toulouse, Laboratoire de biochimie, Institut Fédératif de Biologie de Purpan,

Toulouse, France
An alternative approach would be to upregulate the mitochondrial capacity of the adipocyte. We review proofof-concept studies in which the phenotype of the white adipocyte has been changed to resemble that of the brown adipocyte by expression of peroxisome proliferator-activated receptor coactivator- $1 \alpha$. This increases oxidative capacity and also leads to fatty acid retention through upregulation of glycerol-3-phosphate production, and hence increased fatty acid re-esterification. We conclude that prevention or treatment of insulin resistance through alteration of adipocyte fatty acid handling will require more than a simple alteration of the activity of mitochondrial $\beta$-oxidation within normal limits.

Keywords Adipocytes $\cdot$ Fatty acids $\cdot$ Mitochondria . PGC- $1 \alpha \cdot$ Uncoupling

\section{Abbreviations \\ PGC-1 $\alpha$ peroxisome proliferator-activated receptor coactivator $-1 \alpha$}

\section{Introduction}

In their article in Diabetologia, Maassen et al. [1] suggest the interesting hypothesis that mitochondrial uncoupling in adipose tissue might allow fatty acid oxidation to proceed, thus limiting fatty acid release from adipocytes.

Implicit in their hypothesis is the idea that the release of fatty acids from adipocytes is normally regulated by the balance between fat mobilisation and mitochondrial fatty acid oxidation. We feel that the evidence does not support this idea, and without this it is difficult to believe that upregulation of adipocyte fatty acid oxidation would reduce plasma 
NEFA concentrations, unless the intrinsic capacity of the cells for fatty acid oxidation could be substantially altered.

The pathway of lipolysis and the alternative fates of the fatty acids released in this process are shown schematically in Fig. 1. It is evident that there are several potential control points. Lipolysis itself is highly regulated as is well understood, and is most active in the fasted state and during exercise [2]. But the release of fatty acids from the adipocyte is also regulated beyond the lipolytic step. It is usually considered that re-esterification is the major pathway retaining 'excess' fatty acids within the adipocyte. Maassen et al. [1] are suggesting that fatty acid oxidation within the adipocyte might also restrain fatty acid release.

Oxidative capacity of human white adipose tissue

There is considerable evidence that fatty acid oxidation is not a major pathway in white adipocytes, rodent or human. A number of measurements of the respiratory quotient - the ratio of the rate of carbon dioxide evolution to rate of $\mathrm{O}_{2}$ uptake - of adipose tissue or adipocytes in vitro show this to be close to 1.0, implying exclusive oxidation of glucose, provided that glucose is supplied in the medium (reviewed in [3]). Direct measurements of the rate of fatty acid oxidation in rat adipose tissue show that this is a minor pathway (about $1 \%$ of the activity) compared with fatty acid esterification, the normally-accepted mechanism for preventing release of excess fatty acids delivered from lipolysis [4, 5]. More recent measurements in isolated rat adipocytes confirm the low activity of the oxidation pathway, showing that fatty acid oxidation accounts for approximately $0.2 \%$ of the total fatty acids released in lipolysis; the remainder are distributed almost equally between release and re-esterification [6].

Maassen et al. [1] describe white adipocytes as containing 'large amounts of mitochondria in their tiny cytosolic compartment'. This may be so, but it is important to recognise that white adipose tissue is not a major $\mathrm{O}_{2}$ consuming tissue and that fatty acid oxidation is an $\mathrm{O}_{2}$ demanding process. $\mathrm{O}_{2}$ consumption per $\mathrm{kg}$ wet weight in white adipose tissue is about one-tenth that of resting skeletal muscle (reviewed in [7]), and this is not much altered in obesity or insulin resistance.

These data immediately give a clue as to relative rates of fatty acid oxidation in the two tissues. In the postabsorptive state, adipose tissue supplies fatty acids that form most (around 95\%) of the oxidative fuel for skeletal muscle [8]. If we consider someone with similar amounts of adipose tissue and skeletal muscle (say, $30 \mathrm{~kg}$ of each), then skeletal muscle $\mathrm{O}_{2}$ consumption will be ten times that of adipose tissue (as above). Given that almost all muscle $\mathrm{O}_{2}$ consumption reflects fatty acid oxidation, it is clear that even if all the $\mathrm{O}_{2}$ consumption of white adipose tissue were arising from fatty acid oxidation, this would control less than one-tenth of the flow of fatty acids in the tissue. Healthy people usually have less adipose tissue than muscle, and so adipocyte fatty acid fluxes are quantitatively many times greater than even the highest possible rates of fatty acid oxidation in the tissue.

In the overnight fasted (postabsorptive) state, the ratio of fatty acid release:glycerol release from human adipose tissue is generally close to 3:1. For instance, Samra et al. reported a figure of $3.00 \pm 0.71$ in studies of human adipose tissue in vivo during 14-20 h of fasting, when net fatty acid release from adipose tissue was $1.93 \mu \mathrm{mol} \mathrm{min} \mathrm{m}^{-1}[100 \mathrm{~g}$ tissue $]^{-1}$ (data taken from [9]). This implies that neither fatty acid re-esterification nor fatty acid oxidation normally regulate fatty acid delivery to a detectable extent in human adipose tissue after an overnight fast. We have calculated by how much fatty acid release might be reduced if the tissue were switched to fatty acid oxidation. The $\mathrm{O}_{2}$ consumption of human subcutaneous adipose tissue measured in vivo in healthy subjects in the postabsorptive state was $0.87 \mu \mathrm{mol} \mathrm{min}{ }^{-1}[100 \mathrm{~g} \text { tissue }]^{-1}$ [10]. Oxidation a

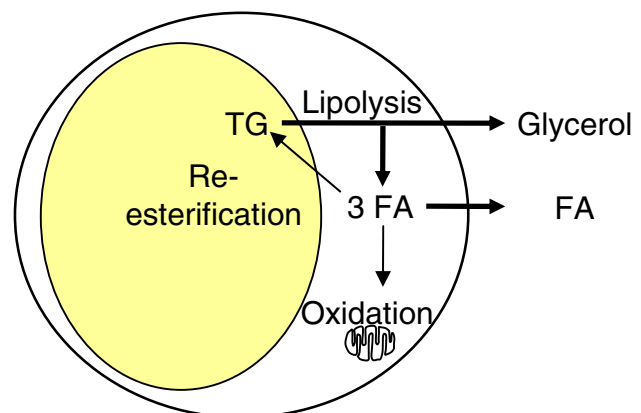

Fig. 1 Schematic pathway for the generation of fatty acids in adipocytes, and their possible fates during stimulation of lipolysis. a In a normal white adipocyte, most of the fatty acids are released b

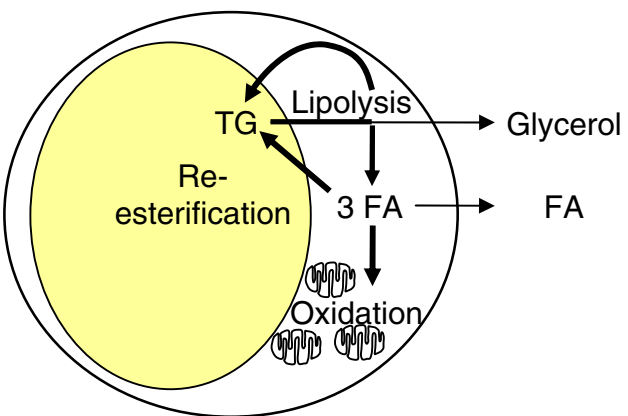

from the cell. b After induction of PGC- $1 \alpha$ expression, mitochondrial biogenesis and upregulation of glycerol kinase activity reduce fatty acid release from the adipocyte. FA, fatty acid; TG, triacylglycerol 
of each mole of fatty acid requires 26.5 moles of $\mathrm{O}_{2}$ (for oleic acid; figures are similar for 'average' fatty acids). If mitochondrial function were altered so that all this $\mathrm{O}_{2}$ consumption were to come from fatty acid oxidation, this would reduce fatty acid release to $1.90 \mu \mathrm{mol} \mathrm{min}^{-1}[100 \mathrm{~g}$ tissue $]^{-1}$, a reduction of $1.7 \%$. These estimates would apply to both lean and obese people [10].

In contrast, in the postprandial period, fatty acid retention in adipose tissue becomes a much more important locus of control. We have used data on $\mathrm{O}_{2}$ consumption and fatty acid metabolism in human white adipose tissue in vivo [10] to assess what might happen in the postprandial state. The data shown in Fig. 2 refer to the $6 \mathrm{~h}$ following a meal. Glycerol release was (median) $120 \mu \mathrm{mol} / 100 \mathrm{~g}$ tissue over $6 \mathrm{~h}$, implying that lipolysis generated $360 \mu \mathrm{mol}$ of fatty acids per $100 \mathrm{~g}$ tissue. $\mathrm{O}_{2}$ consumption over the same period was $330 \mu \mathrm{mol} / 100 \mathrm{~g}$ tissue. As discussed earlier, this $\mathrm{O}_{2}$ consumption probably reflects mainly glucose oxidation. If, by manipulation of uncoupling, for instance, it could be made to represent entirely fatty acid consumption, then it would account for $3.5 \%$ of the rate of fatty acid release from lipolysis. It therefore seems that mitochondrial fatty acid oxidation in white adipose tissue under normal circumstances could control only a few per cent of the rate

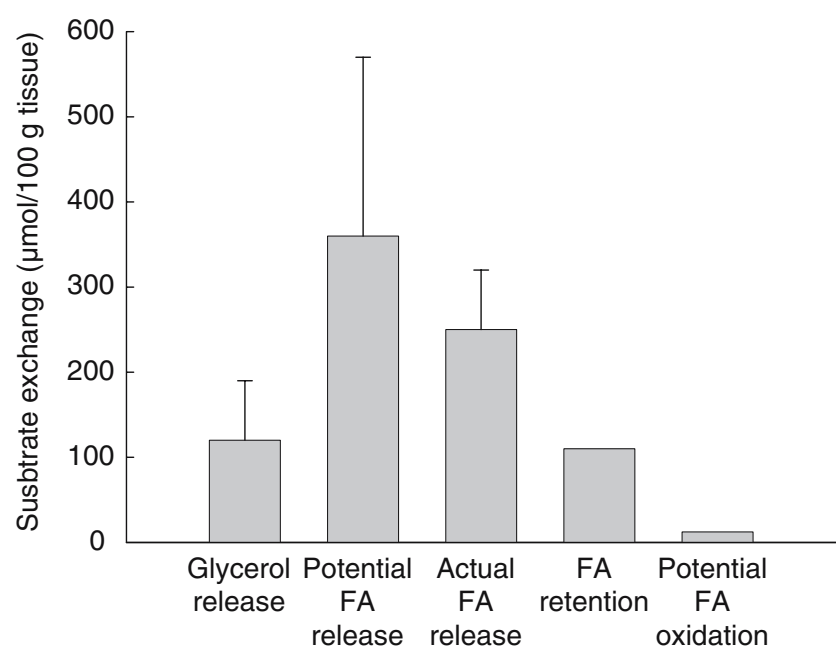

Fig. 2 Measurements of the metabolism of human subcutaneous adipose tissue in vivo in healthy volunteers. The data are taken from Coppack et al. [10]. They represent integrated fluxes, starting in the postabsorptive state and then followed for $6 \mathrm{~h}$ after a mixed meal. All are expressed as median values, in $\mu \mathrm{mol} / 100 \mathrm{~g}$ tissue. Measured data are shown with upper end of interquartile range. Glycerol release and fatty acid (FA) release from the tissue were measured. Potential FA release is glycerol release $\times 3$, assuming that glycerol release reflects triacylglycerol hydrolysis. FA retention is the amount of fatty acid retained (potential minus actual FA release). Potential FA oxidation is the amount of fatty acid that could have been oxidised if all the (measured) $\mathrm{O}_{2}$ consumption of the tissue reflected FA oxidation, which, as described in the text, is highly unlikely of fatty acid release from adipocytes. It is possible that this could be increased two- to threefold through mitochondrial uncoupling, although even then a metabolic switch would be needed to make fatty acids the predominant substrate. The data suggest, however, that the ability to oxidise fatty acids is intrinsically limited by the mitochondrial capacity of white adipocytes.

In fact, these data emphasise that under normal circumstances, the major route of retention of fatty acids in the adipocyte must be re-esterification. We know that reesterification is a highly regulated process, controlled by insulin and possibly other factors [11], and this pathway might present itself as a more attractive target for pharmacological intervention. Indeed, there is evidence that this is the very pathway targeted by the thiazolidinedione insulin-sensitising agents [12].

Increasing the oxidative capacity of white adipose tissue: alternative strategies

The only way in which this situation might be substantially altered is if the intrinsic oxidative capacity of adipose tissue could be increased. Clearly, in brown adipose tissue, the rate of fatty acid oxidation is of a different order of magnitude to the rate of lipolysis. In brown adipocytes, most of the fatty acids released in lipolysis are indeed consumed by uncoupled mitochondrial fatty acid oxidation. This raises the question of whether it is possible to transform the phenotype of the white adipocyte to be more like a brown fat cell. As a proof of concept, we have expressed the gene encoding the transcriptional coactivator PGC $-1 \alpha$ in human white adipocytes $[13,14]$. PGC- $1 \alpha$ switches on genes involved in fatty acid oxidation and the mitochondrial respiratory chain. Moreover, ectopic expression of the coactivator gene induces the expression of the uncoupling protein 1 gene, which encodes a mitochondrial inner membrane protein that uncouples $\mathrm{O}_{2}$ consumption from ATP synthesis. The coordinated upregulation of gene expression results in an increased capacity to oxidise fatty acids. Moreover, an induction of glycerol kinase which is expressed at low levels in white fat is also observed. The enzyme catalyses the phosphorylation of glycerol to form glycerol-3-phosphate, which constitutes the backbone of triacylglycerol. Glycerol kinase is therefore involved in fatty acid esterification. If the cells are stimulated by catecholamines, an increased glycerol kinase activity generates a futile cycle through the direct reincorporation into triacylglycerols of glycerol and fatty acids resulting from triacylglycerol hydrolysis. Therefore, turning on the expression of the PGC- $1 \alpha$ gene and increasing its protein production may favour the use of fatty acids within the fat cell instead of a release into the bloodstream. 


\section{Conclusions}

We conclude that prevention or treatment of insulin resistance through modification of adipocyte fatty acid handling will require more than a simple alteration of the activity of mitochondrial $\beta$-oxidation. It might be possible to increase the total capacity for fatty acid oxidation by promoting the conversion of white adipocytes towards a brown adipocyte phenotype [15]. Otherwise, it is clear that a more attractive target pathway for restraining fatty acid release would be that of fatty acid esterification [16].

Acknowledgements The authors collaborate within the project 'Hepatic and adipose tissue and functions in the metabolic syndrome' (HEPADIP, http://www.hepadip.org/), which is supported by the European Commission as an Integrated Project under the 6th Framework Programme (Contract LSHM-CT-2005-018734). We thank M. Brand, Medical Research Council Dunn Human Nutrition Unit, Cambridge, for information on mitochondrial $\mathrm{O}_{2}$ consumption.

Duality of interest The authors declare that there is no duality of interest associated with this manuscript.

\section{References}

1. Maassen JA, Romijn JA, Heine RJ (2007) Fatty acid-induced mitochondrial uncoupling in adipocytes as a key protective factor against insulin resistance and beta cell dysfunction: a new concept in the pathogenesis of obesity-associated type 2 diabetes mellitus. Diabetologia 50:2036-2041

2. Frayn KN, Langin D (2004) Triacylglycerol metabolism in adipose tissue. In: van der Vusse GJ (ed) Lipobiology. Elsevier, Amsterdam, pp 337-356

3. Frayn KN (1992) Studies of human adipose tissue in vivo. In: Kinney JM, Tucker HN (eds) Energy metabolism: tissue determinants and cellular corollaries. Raven, New York, pp 267-295
4. Harper RD, Saggerson ED (1976) Factors affecting fatty acid oxidation in fat cells isolated from rat white adipose tissue. J Lipid Res 17:516-526

5. Baht HS, Saggerson ED (1988) Comparison of triacylglycerol synthesis in rat brown and white adipocytes. Effects of hypothyroidism and streptozotocin-diabetes on enzyme activities and metabolic fluxes. Biochem J 250:325-333

6. Wang T, Zang Y, Ling W, Corkey BE, Guo W (2003) Metabolic partitioning of endogenous fatty acid in adipocytes. Obes Res $11: 880-887$

7. Frayn KN, Humphreys SM, Coppack SW (1995) Fuel selection in white adipose tissue. Proc Nutr Soc 54:177-189

8. Andres R, Cader G, Zierler KL (1956) The quantitatively minor role of carbohydrate in oxidative metabolism by skeletal muscle in intact man in the basal state. Measurements of oxygen and glucose uptake and carbon dioxide and lactate production in the forearm. J Clin Invest 35:671-682

9. Samra JS, Clark ML, Humphreys SM, Macdonald IA, Frayn KN (1996) Regulation of lipid metabolism in adipose tissue during early starvation. Am J Physiol 271:E541-E546

10. Coppack SW, Fisher RM, Gibbons GF et al (1990) Postprandial substrate deposition in human forearm and adipose tissues in vivo. Clin Sci 79:339-348

11. Evans K, Burdge GC, Wootton SA, Clark ML, Frayn KN (2002) Regulation of dietary fatty acid entrapment in subcutaneous adipose tissue and skeletal muscle. Diabetes 51:2684-2690

12. Oakes ND, Thalén PG, Jacinto SM, Ljung B (2001) Thiazolidinediones increase plasma-adipose tissue FFA exchange capacity and enhance insulin-mediated control of systemic FFA availability. Diabetes 50:1158-1165

13. Tiraby C, Tavernier G, Lefort C et al (2003) Acquirement of brown fat cell features by human white adipocytes. J Biol Chem 278:33370-33376

14. Mazzucotelli A, Viguerie N, Tiraby C et al (2007) The transcriptional coactivator peroxisome proliferator activated receptor (PPAR) $\gamma$ coactivator- $1 \alpha$ and the nuclear receptor PPAR $\alpha$ control the expression of glycerol kinase and metabolism genes independently of PPAR $\gamma$ activation in human white adipocytes. Diabetes 56:2467-2475

15. Tiraby C, Langin D (2003) Conversion from white to brown adipocytes: a strategy for the control of fat mass. Trends Endocrinol Metab 14:439-441

16. Frayn KN (2002) Adipose tissue as a buffer for daily lipid flux. Diabetologia 45:1201-1210 